\title{
Should pluralists be pluralists about pluralism?
}

\author{
Robert Passmann ${ }^{1,2}$ (iD
}

Received: 21 May 2021 / Accepted: 3 August 2021 / Published online: 10 August 2021

(c) The Author(s) 2021

\begin{abstract}
How many correct logics are there? Monists endorse that there is one, pluralists argue for many, and nihilists claim that there are none. Reasoning about these views requires a logic. That is the meta-logic. It turns out that there are some meta-logical challenges specifically for the pluralists. I will argue that these depend on an implicitly assumed absoluteness of correct logic. Pluralists can solve the challenges by giving up on this absoluteness and instead adopt contextualism about correct logic. This contextualism is naturalistically appealing.
\end{abstract}

Keywords Logical pluralism · Correct logic · Meta-logic · Contextualism • Meaning-variance $\cdot$ Coherence

\section{Introduction}

Which logic(s) can be considered correct, right, adequate, or true? Philosophers of logic usually require that a logic must, at the very least, correctly codify 'our' pretheoretic notion of logical consequence to earn such a predicate. What this means exactly is a highly contentious issue. Different views on this question can, however, be classified according to how many logics they deem true or correct: monists endorse that there is one, pluralists argue for many, and nihilists claim that there are none. Logical pluralism, i.e. the view that there are at least two correct logics, has been heavily discussed in recent years (e.g., Priest 2001; Beall and Restall 2005; Read 2006; Shapiro 2014; Caret 2017; Steinberger 2019). ${ }^{1}$

\footnotetext{
1 Throughout this paper, I will use the adjective 'correct' to denote those logics that correctly capture the notion of logical consequence, in some sense to be specified. 'Correct logic' should therefore be read synonymously with 'true logic' and similar phrases.

$\triangle$ Robert Passmann

r.passmann@uva.nl

1 Institute for Logic, Language and Computation (ILLC), University of Amsterdam, Amsterdam, The Netherlands

2 St John's College, University of Cambridge, Cambridge, UK
} 
Reasoning about these views requires a logic - the meta-logic. Despite this fact, meta-logical considerations, in particular in relation to logical pluralism, have received only sparse attention. Sereni and Fogliani (2017) studied the pluralist's meta-logical options and were left with many concerns that pluralists must account for 'if a sufficient degree of plausibility is to be provided to any version of logical pluralism' (p. 367). To my knowledge, a pluralist reply to their concerns has been missing until now. Moreover, Griffiths and Paseau (2021) have recently argued that logical pluralism faces problems with respect to meta-logical reasoning. I will argue that these problems depend on an implicitly assumed absoluteness of correct logic, i.e. the claim that it is independent of the meta-logic whether or not a given logic is correct.

In this paper, I propose a solution that pluralists can adopt to defend their views against these objections: a contextualist understanding of 'correct logic'. For the contextualist, it is not an absolute fact whether a given logic is correct or not. Instead, whether or not a logic is correct depends explicitly on the meta-logical context. If a pluralist adopts contextualism about correct logic, then they will (most likely) endorse different sets of correct logics, depending on the meta-logic. This contextualism does not only solve several worries about the meta-logic of logical pluralism but is also a better explanation of certain data from (mathematical) logic. It is therefore a naturalistically appealing extension of logical pluralism in that it takes mathematical facts seriously.

Overview I will briefly recall in section 2 how to achieve proper logical pluralism. Section 3 will then be concerned with different conceptions of correct logic. Section 4 will deal with the meta-logic employed by different conceptions of correct logic. In particular, I will discuss two worries one could have with respect to the meta-logic of pluralist conceptions. Finally, in section 5, I suggest that a form of contextualism allows pluralists to solve these issues, and is naturalistically appealing.

\section{Pluralism proper}

There are different ways of endorsing many logics but not all result in pluralism proper. In a nutshell: logical pluralists want proper disagreement about the validity of an argument. I will briefly discuss how to achieve this, or fail to. Note that I identify logics with their consequence relations in this paper.

Pluralism proper cannot arise due to syntactical choices. For example, endorsing two versions of intuitionistic logic, one with Roman and one with Greek letters, is not pluralism. Furthermore, pluralism proper does not arise from endorsing two logics that agree where they overlap, such as classical propositional and first-order logic. If each logic has its unique domain of application, then no disagreement about the validity of an argument can arise as each argument belongs to a domain. These domain-specific logics can be combined into a global consequence relation of unrestricted domain. So there is no pluralism proper unless we require at least two logics with the same or unrestricted domains of application. ${ }^{2}$

\footnotetext{
2 Steinberger (2019, Section 3) gives a comprehensive analysis of different forms of pluralism; see also Russell (2021).
} 
Pluralism should not arise due to a Quinean (1986, pp. 80-81) meaning-variance of the logical language. If intuitionists talk about 'not ${ }_{i}$ ' (' $\neg$ ') and classicists about ' not $_{\mathrm{c}}$ ' (' $\neg$ '), then a unique notion of logical consequence can account for why an

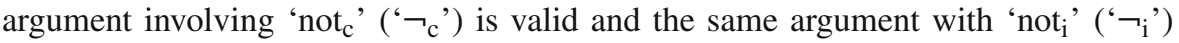
is not. Intuitionists use the intuitionistic language, classicists the classical language. Pluralism proper, however, fixes the logical language and endorses distinct notions of logical consequence. Hjortland (2013) discusses different forms of meaning-variance in detail. I will discuss meaning-variance at the level of meta-logic in section 4.2.

Finally, many pluralists demand a naturalistic approach that takes logical practice and mathematical results about logics seriously. Shapiro's (2014) pluralism is developed from such a naturalistic perspective. Caret argues more generally for naturalising the debate between monists and pluralists:

An honest naturalist simply takes mathematics as it stands and respects the autonomy of the discipline, rather than imposing outside ideas about how it 'should' be practiced. Who are we to police the bounds of mathematics because of some hangup about bivalence or truth-tables? (2019, p. 17)

Such naturalistic positions about logic can be seen as part of the larger debate surrounding anti-exceptionalism about logic. ${ }^{3}$

Pluralism proper meets these criteria and leaves at least two correct logics. I will discuss what makes a logic correct in the next section.

\section{Conceptions of correct logic}

In this section, I will illustrate some positions in the debate on monism, pluralism, and nihilism. I will also introduce an abstract notion of conceptions of correct logic.

The pluralism of Beall and Restall (2005) will be my running example. They submit that correct logics arise through the Generalised Tarski Thesis (GTT):

Generalised Tarski Thesis (GTT). An argument is valid if $_{\mathrm{x}}$ and only if, in every case $_{\mathrm{x}}$ in which the premises are true, so is the conclusion. $(2005$, p. 29)

A specification of cases is admissible if and only if the resulting instance of the GTT gives rise to a logic that is necessary, normative and formal. A correct logic is one that arises through an admissible specification of cases in the GTT. Beall and Restall show that several instances of cases are admissible, and conclude that at least classical, intuitionistic and relevant logics are correct. Beall-Restall-pluralism has been extensively discussed in the literature and faces several problems in its original formulation. ${ }^{4}$ I will use this view as my running example because it is probably the most well-known view on logical pluralism, precisely enough formulated and at the same time simple enough to get things going. Still, I use their pluralism only as an example; it is not a view I am arguing for.

\footnotetext{
3 See, for example, Hjortland (2013), Martin and Hjortland (2021) and Priest (2014).

4 See, for example, Priest (2001, 2005), Read (2006), Griffiths (2013) and Keefe (2014).
} 
Another example is Shapiro's (2014) 'eclectic pluralism' endorsing all logics that have technical interest or fruitful applications in mathematics. Well-known monists are Quine (1986) with classical logic, Dummett (1975) arguing that intuitionistic logic is the only correct logic, and Read (2006), who endorses relevance logic.

A nihilist conception is discussed by Russell (2018), who provides counterexamples for even the most basic logical rules, such as the identity rule or disjunction introduction, e.g. by defining an atomic proposition 'PREM' that is true if and only if it appears in a premise. Then, 'PREM; therefore PREM' is a counterexample to the identity rule. She derives the nihilist conclusion that there is no logic with unrestricted domain of application. Russell's reply to nihilism is 'lemma incorporation', that is, recognising the hidden assumptions of logical laws such as bivalence as a condition for the law of excluded middle.

All these conceptions of correct logic have in common that they specify the nature of correct logic in terms of certain desiderata $P_{1}, P_{2}, P_{3}, \ldots$ and so forth. A logic is correct if and only if it satisfies the desiderata specified by the conception in question. Such desiderata could be $\left(P_{1}\right)$ axiomatisability, $\left(P_{2}\right)$ decidability, $\left(P_{3}\right)$ the existence of good semantics, $\left(P_{4}\right)$ truth-preservation, or $\left(P_{5}\right)$ formality. Paseau $(2007$, p. 393) further suggests $\left(P_{6}\right)$ strength, $\left(P_{7}\right)$ the best suitability for mathematical reasoning, and $\left(P_{8}\right)$ the best ability to model natural language phenomena. Beall-Restall-pluralism uses $\left(P_{9}\right)$ formality, $\left(P_{10}\right)$ necessity, and $\left(P_{11}\right)$ normativity. Each conception specifies a selection of desiderata; the desiderata mentioned here are just arbitrarily chosen examples. The stronger a conception's desiderata are, the fewer logics it will deem correct. The more logics a conception deems correct, the more permissive its desiderata must be.

To summarise, a conception specifies desiderata a logic must satisfy to be correct, and endorses a (possibly empty) collection of correct logics. The conception is nihilist if there is no logic satisfying the $P_{i}$, monist if there is exactly one, and pluralist if there are many such logics.

\section{Meta-logic}

In this section, I focus on the use of meta-logic in the debate on logical pluralism. First, I will show that meta-logic matters and that we cannot do without it. I will then discuss two meta-logical worries for the pluralist: the meaning-variance-worry and the coherence-worry.

\subsection{Meta-logic matters}

Meta-logic plays a crucial role in discussing logics, even if the main argument for a particular conception is non-deductive. Most desiderata involve abstract properties of logics. Usually, a deductive proof is needed to show that a logic satisfies a desideratum; certainly so for desiderata such as 'consistency' or 'axiomatisability'. 5 Griffiths and

\footnotetext{
5 Even if you employ non-deductive argumentation standards, meta-logic is required to sensibly discuss technical desiderata such as 'Kripke-completeness' or 'axiomatisability'. As we will see below, the truth
} 
Paseau (2021) discuss the need for a deductive meta-logic even in the case of an abductive argument for pluralism. Woods (2019) shows that abductive arguments about logic require a deductive background. ${ }^{6}$

Often, there is a need for elaborate mathematical tools such as the possible worlds semantics for intuitionistic logic, i.e. Kripke models. These tools are usually considered within a set-theoretic background theory. For probabilistic or infinitary logics, we need real numbers and infinite cardinals, which depend heavily on the precise metatheory. The infinite cardinals might not be linearly ordered without the axiom of choice, and different constructions of the real numbers might not coincide in constructive set theory. ${ }^{7}$ Even seemingly simple matters depend on the meta-theory. Shapiro (2014, pp. 194-199) shows that the choice of meta-logic may influence the properties of a fixed object-logic, e.g. by presenting an argument whose validity in intuitionistic objectlogic depends on the meta-logic permitting a certain instance of excluded middle. He also points out that the 'consistency of a theory' can depend on the meta-theory. A theory that is consistent in a given meta-logic might be inconsistent in another (Shapiro 2014, p. 166). All this shows that it is impossible to exclude meta-mathematics from the debate.

Dummett (1975 ,pp. 29-31) discusses the need for a 'common ground' in the debate between classical and intuitionistic logicians populated by 'auxiliary statements' about whose interpretation both sides agree. This is an external dialectic perspective: do the meta-logical commitments of the conceptions leave enough room-'common ground'- to discuss them in a way that does not beg the question and, possibly, convince others? Or is this hindered by strong meta-logical requirements? There seems to be no reason to demand differently of different conceptions: they should all be held to the same standard, evaluated on a 'common ground' as far as possible.

In conclusion, meta-logic has a central role in every argument for or against conceptions of correct logics, i.e. the object-logics of the conception in question, even if these arguments are not entirely deductive. For the remainder of this paper, I will focus on two meta-logical problems that are internal to pluralist conceptions. Let's have a look.

\subsection{The meaning-variance-worry}

Pluralists frequently suggest the use of more than one meta-logic. Shapiro endorses that '[f]or the eclectic logician, any of the established logics can be used to prove

\footnotetext{
Footnote 5 continued

(and meaning) of such desiderata might change with meta-logic: for example, when someone points out that 'intuitionistic logic is Kripke-complete', then they implicitly commit to a classical meta-logic because intuitionistic metatheories cannot prove the Kripke-completeness of intuitionistic logic; see below and McCarty (2008). This is the way in which meta-logic is crucial for the present debate. I do not claim that all standards of meta-level reasoning depend on the meta-logic. I thank a referee for pushing me to clarify this point.

6 This discussion is led under the headings of 'the background logic problem' or 'the centrality problem', see also Shapiro (2000) and Wright (1986).

7 Jech (1966) showed that every partial ordering can be embedded into the cardinals of a model of ZF-set theory. Lubarsky and Rathjen (2008) proved that it is consistent with constructive CZF-set theory that the Cauchy reals are a set while the Dedekind reals are a proper class.
} 
metatheoretic results about any of the established logics' (2014, p. 204). Similarly, Beall and Restall want their 'own reasoning to be evaluated by [...] any and all (admissible) [consequence relations]' (2005, p. 99).

I discussed that pluralism proper does not arise through meaning-variance in the logical language, e.g. the intuitionist's 'or' meaning something different than the classicist's 'or'. However, pluralists endorse a meaning-variance of meta-logical expressions such as 'valid' (for a full discussion see Hjortland 2013). This meaning-variance happens within a fixed meta-logic and the meaning depends on the object-logics. For instance, if the pluralist endorses two logics $\mathcal{L}_{1}$ and $\mathcal{L}_{2}$ that disagree on the validity of an argument $P \vdash Q$, then the disagreement should arise from the fact that the meaning of validity in $\mathcal{L}_{1}$ and $\mathcal{L}_{2}$ diverge. The disagreement should not arise because the meaning of the logical constants appearing in $P$ or $Q$ depends on the object logics.

Now, with considerations of meta-logic, there is possibly another level of meaningvariance: among the meta-logics. Suppose that a conception of correct logic stipulates some desiderata that a logic has to satisfy to be correct. If the pluralist allows many meta-logics, then they run into the risk of varying the meaning of their desiderata. Take Shapiro's 'consistency'. We saw that its extension - those logics that are consistentvaries across meta-logic, and one might worry that its intension does as well. After all, consistency-in-intuitionistic-meta-logic is different from consistency-in-classicalmeta-logic in that the former requires a constructive proof of consistency and the latter a classical one, i.e. the former is a stronger requirement than the latter.

Let's consider another possible desideratum that a conception of correct logic could employ: a logic is called Kripke-complete if and only if there is a class of Kripke models characterising that logic. It is well-known that classical logic, intuitionistic logic and many intermediate logics are Kripke-complete. ${ }^{8}$ Results of McCarty, which I will also consider below, show that the Kripke-completeness of intuitionistic logic requires the full law of excluded middle. In fact, it turns out that there are intuitionistic metatheories in which 'the relevant completeness theorems are not just underivable in those metatheories but provably false' (McCarty 2008, p. 1316). These results illustrate that the extension of 'Kripke-complete' is not absolute but depends on the meta-logic (and meta-theory).

In this situation, one might worry that not only the extension of 'Kripke-complete logic' varies but also its intension. McCarty's results entail that certain Kripke-models which exist in classical metatheories do provably not exist in those intuitionistic metatheories in which the completeness theorems fail. In this situation, can we still say that Kripke-completeness-in-intuitionistic-metatheories and Kripke-completeness-inclassical-metatheories have the same intension? I think it is conceivable that they do not. The properties of 'completeness w.r.t. Tarski models' and 'completeness w.r.t. many-valued models' do not have the same intension because they refer to two distinct classes of models. Exactly the same happens when we consider 'Kripke-completeness' in different meta-theories: both its extension and intension change.

This example illustrates that the meaning of the desiderata can change in virtue of meta-logic. But if the desiderata change meaning across meta-logic, then 'correct logic' will do so as well because its meaning is given by the desiderata. With many

\footnotetext{
8 Not all intermediate predicate logics are Kripke-complete; e.g. Skvortsov (1998).
} 
meta-logics, there might thus be many meanings of 'correct logic'. But which one is then the intended meaning of 'correct logic'? That is the meta-logical meaningvariance-worry.

If the intended meaning of 'correct logic' can only be found under a certain metalogic, then only that meta-logic can be used to give a meta-logical analysis of logical pluralism - but as mentioned above, pluralists like Shapiro as well as Beall and Restall endorse the use of more than one meta-logic. To make sure that such analyses are meaningful, we require a certain degree of meaning-invariance across meta-logics to make sure that one and the same concept of correct logic is discussed. Even if the pluralist conception at hand has successfully established that there is no meaningvariance amongst the logical language of the object-logics, it is not clear that this meaning-invariance transfers to the level of meta-logic.

I will show that a contextualist understanding of 'correct logic' across meta-logics will allow the pluralist to dispel any worries about meta-logical meaning-variance. Note that the meta-logical meaning-variance worry might be more or less grave depending on the particular conception of correct logic at hand. If a conception endorses desiderata which are sufficiently stable under changes of meta-logic to not worry about their meaning-variance, then this conception will not suffer from this worry. The specific nature of the contextualism I am about to propose will depend on whether or not the meaning-variance worry is serious for a given conception. Before discussing this, I consider another worry in the next section.

\subsection{The coherence-worry}

I will say that a conception is coherent if its meta-logic is justified with respect to the logics that the conception endorses on the object-level. Coherence is internal to a conception.

Showing the coherence of a monist conception would be straightforward: it suffices to demonstrate that their meta-logical considerations, e.g. the argument for their conception, are valid in their one correct logic.

What about pluralism? According to Griffiths and Paseau (2021), logical pluralism is an unstable position due to meta-logical reasoning. This comes about as follows: recall, for example, that Beall and Restall endorse classical, intuitionistic and relevance logic, and thereby (if they are successful) settle the dispute between classical, intuitionistic and relevance logicians about which of these logic is the right one (namely, all of them). To conduct these arguments, Beall and Restall need a meta-logic. Griffiths and Paseau conclude that the argument for a specific pluralist view must be valid in all logics endorsed. Beall and Restall would need an argument that is valid not only in classical logic but also in intuitionistic logic and relevance logic. The more permissive the pluralist conception, the weaker their tools to prove their argument. In this view, an extremely permissive pluralist conception such as Shapiro's (2014), endorsing all nontrivial logics, will have essentially no meta-logical devices left. According to Griffiths and Paseau, this rules out extremely permissive pluralism and less permissive pluralist conceptions are ruled out for other reasons ${ }^{9}$ as unmotivated.

\footnotetext{
${ }^{9}$ See Griffiths (2013).
} 
Before I continue with an analysis of the assumptions that underlie this meta-logical instability of logical pluralism, let me remark that there is a weak as well as a strong reading of Griffiths and Paseau's conclusion. The strong reading requires that one and the same argument for the pluralist conception must be valid in every single logic the conception endorses. Under the weak reading, there must be an argument with the same conclusion in every single one of the logics endorsed. Griffiths and Paseau initially have the strong reading in mind, and I agree with them that their argument does not suffer much from adopting the weaker reading: instead of finding one argument that works in all logics, the pluralist must now find a valid argument for pluralism in each endorsed logics. In the stronger case, the task is impossible because there is not enough logical force left to develop the argument. But this shows that under the weaker reading, the task cannot be solved by developing a largely uniform proof strategy. So the pluralist would really need to develop many different arguments, tailored to each endorsed logic. This seems practically impossible. ${ }^{10}$

Let's return to the meta-logical instability-argument and its assumptions. This argument can only develop its force from the supposed fact that the pluralists must derive the same conclusion in all the logics they endorse. But is this a natural assumption for the pluralist? I will get to that in section 5. For now, I note that Griffiths and Paseau rely on the following principle (which they do not explicitly endorse).

Absoluteness of correct logic. It is independent of meta-logic whether or not a given logic is a correct logic.

This principle only governs the relation between object-logics and meta-logics of a conception. It does not specify a desideratum that a correct logic has to satisfy.

Sereni and Fogliani (2017) conduct a systematic study of many meta-logical options the pluralist has, such as using the strongest logic, the weakest logic, no logic, or many logics. They conclude that each option comes with its own set of coherence problems. In doing so, they assume that the object-logics of a conception are a fixed determinate collection, e.g. when they talk about 'the intersection of all those (object-)logics which the pluralist accepts' (p. 353) or about 'the strongest among the object-logics' (p. 356). To appreciate that Sereni and Fogliani do indeed rest their analysis on the absoluteness of correct logic, let's have a brief look at their 'toy example' case, that they repeatedly use.

Their toy example is this: a pluralist endorses two object logics, both classical and intuitionistic logic. What meta-logical options do they have? Sereni and Fogliani discuss three scenarios: (i) there are classically and intuitionistically valid arguments showing that intuitionistic logic is a correct logic but only a classical argument showing that classical logic is correct; (ii) there is a single global argument that both logics are correct but this argument is only classically valid; or (iii) there is an argument valid in each of the logics that each of the logics is correct. Assuming the universality of logic,

\footnotetext{
${ }^{10}$ We can imagine a pluralist who endorses intuitionistic logic and all superintuitionistic logics. This pluralist would just need to find a single argument in intuitionistic logic to fulfil Griffiths and Paseau's conclusion. But this means that they must interpret such a pluralist to not fall in the category of "very permissive pluralism' but rather the category of 'unmotivated pluralism', even though this pluralist endorses infinitely-many (in fact, continuum-many) logics. So, Griffiths and Paseau must have something different in mind than just cardinality when they talk about the permissiveness of a pluralist conception.
} 
they find both scenarios (i) and (ii) undesirable because intuitionistic logic cannot do one of the crucial tasks a logic with universal application should be able to do, viz. 'sanctioning' which logics are acceptable and which are not. Leaving aside a discussion of whether or not their assumption of universality is innocent, this criticism is clearly based on the assumption that the truth of a given (object-)logic is a fact, independent of the choice of meta-logic. If they did not endorse the absoluteness of correct logic, they would not have anything to object here.

Sereni and Fogliani's treatment of option (iii) leads to a different problem. In the example above, they deny that option (iii) leads to a genuine meta-theoretic pluralism because 'a pluralist of this kind would just have two classically valid arguments for [logical pluralism]' (p. 366). While they are, of course, right that every intuitionistically valid argument is also classically valid, I do not agree that the result must be ingenuine pluralism. There is no coherence issue as in cases (i) and (ii), even on the assumption of absoluteness.

They also take the third case to illustrate a 'regress of pluralisms' (p. 367), as an instance of a regress argument that they spell out in different forms throughout their article: how are the object-logics justified? In virtue of some meta-logic(s). But how are those justified? Well, that must be due to some meta-meta-logic(s), and so on, ad infinitum. Two remarks are in order. First, I do not think that this regress is a challenge that applies only to the pluralist. All participants of the debate must justify their metatheoretic reasoning whether or not they endorse many, one or no logics. Second, if Sereni and Fogliani indeed, as it seems, endorse the universality of logic, then there is no problem here because if a logic is universal, then it may certainly be used for meta-theoretic purposes.

In conclusion, it turns out that Sereni and Fogliani as well as Griffiths and Paseau assume the absoluteness of correct logic in their attacks on the meta-logical coherence of pluralist conceptions.

Before moving on to the next section, let me briefly remark that nihilist conceptions face analogous meta-logical challenges: first, note that the nihilist is likely to accept absoluteness. Proper nihilism endorses that there is no correct logic and this should be independent of the meta-logic. Russell's (2018) argument for logical nihilism seems to employ a classical meta-logic. But how can that be justified when there is no correct logic? The nihilist could argue that the validity of a logical rule is bivalent, i.e. a logical rule must either be valid or not valid. I would not be convinced as this seems to be a retreat into domain-specific improper pluralism.

\section{Contextualism}

In this section, I will first produce some evidence from logic and then let naturalism be the judge. The result will be a contextualist understanding of 'correct logic' with respect to meta-logic. 


\subsection{A case study}

I mentioned above that many pluralists favour naturalistic approaches. Caret argued that we should take 'mathematics as it stands and respect the autonomy of the discipline' (2019, p. 17). I will follow this maxim now by conducting a brief case-study on how 'correct logic' depends on the meta-logic.

Consider again Beall-Restall-pluralism. They argue that at least classical, intuitionistic and relevance logics satisfy their desiderata. Intuitionistic logic is obtained as correct by instantiating cases $_{\mathrm{x}}$ with 'systems of stages', i.e. Kripke models (2005, pp. 62-64). For these 'systems of stages' to give rise to intuitionistic logical consequence, one needs a completeness theorem stating that: if a certain inference is valid in all 'systems of stages', then it is a valid inference of intuitionistic logic. After all, they obtain intuitionistic logic as the logic arising from such Kripke models. It turns out, however, that such a result is problematic in a purely intuitionistic meta-theory. To appreciate this, we have to dive into some mathematics.

McCarty (2008), generalising a theorem of Kreisel (1962) and Gödel, ${ }^{11}$ proved that a completeness theorem for Kripke models, as desired above, requires logical principles that are not purely intuitionistic:

HA [Heyting Arithmetic] and its extensions show that, if intuitionistic predicate logic is weakly complete with respect to Tarski, Beth or Kripke semantics, then MP [Markov's Principle] holds. (McCarty 2008, p. 1326, Corollary 11)

Dummett points out that 'Markov's principle is definitely incorrect intuitionistically, since it is inconsistent with the theory of lawless sequences; it can also be shown to be inconsistent with the theory of the creative subject' (1977, p. 175). Moreover, Markov's principle is 'demonstrably underivable either in the system HA of intuitionistic arithmetic or in the standard systems of intuitionistic analysis' (p. 175). So, asserting the Kripke completeness of intuitionistic logic in the setting of an intuitionistic meta-theory is both philosophically and mathematically contentious.

Of course, we need neither be committed to Brouwerian intuitionism nor to Heyting's artihmetic HA for our meta-theory. That is certainly true but the trouble does not stop here. Moving to a different meta-theory can be problematic as well because McCarty's results entail that 'any extension of HAA [intuitionistic second-order arithmetic with intuitionistic comprehension], HAS [intuitionistic second-order arithmetic] or IZF [intuitionistic Zermelo-Fraenkel set theory] that proves the completeness of propositional or predicate intuitionistic logic with respect to ... Kripke semantics is classical' (2008, p. 1323, Corollary 5). So whenever we work in any of these meta-theories then intuitionistic logic can only be Kripke-complete if we are actually working in a classical meta-theory. But that is certainly undesirable if our goal is to assess whether Beall and Restall's argument is intuitionistically valid. All this suggests that Kripke models are 'only [a] classical means to study intuitionistic logic, for

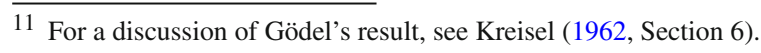


it can be shown that an intuitionistic completeness proof with respect to them cannot exist' (Iemhoff 2020). ${ }^{12}$

It might still be possible to make Beall and Restall's argument work: there might be a meta-theory that can be justified as intuitionistic and allows for the Kripke completeness of intuitionistic logic, or it might be possible to replace Kripke models with a different semantics. ${ }^{13}$ The point I want to make here is that the question of whether or not Beall and Restall's argument is intuitionistically valid is a difficult question whose answer depends on specific meta-mathematical commitments. For now, I conclude that with a move from classical to intuitionistic meta-logic, the argument that Beall and Restall give in favour of intuitionistic logic being a correct logic cannot be assumed valid: whether or not the resulting meta-theory can show that the GTT, instantiated with 'systems of stages', gives rise to intuitionistic logic requires more work. $^{14}$

Williamson (2014) concludes from part of the mathematical data I used above that 'the dispute between classical and intuitionistic logic in the object language, far from being resolved by metalogic, is reproduced in the metalanguage' (p. 218) as an instance of his more general point that meta-logic is not a 'neutral arbiter'. I agree. Meta-logic takes a stance.

Beall and Restall deliberately do not specify the meta-logic of their reasoning (2005, p. 99). Read (2006), however, ascribes a classical meta-logic to Beall-Restallpluralism because of their reliance on classical semantics. He criticises classical metalogic as 'insensitive to the real nature' (p. 205) of non-classical logics. In Read's view, relevance logic is the only correct logic, and he therefore criticises Beall and Restall's reliance on classical meta-logic as 'a strange approach to take, if one believes relevance logic is the correct logic. Why use an alien logic for one's metatheory-and if one does, why trust the result?' (p. 208). Read argues that Beall and Restall's desiderata must be 'interpreted, and developed, in a relevant metalanguage in which the relevance of the premises to the conclusion is an integral part of truth-preservation' (p. 209). He proceeds as follows: the GTT defines validity in terms of a conditional, 'in every case $_{\mathrm{x}}$ in which the premises are true, so is the conclusion'. According to Read, this conditional must not be interpreted as a material but a relevance implication because the former does not result in a correct notion of validity in a relevance meta-logic. He concludes that the GTT will then only give rise to relevance validity. That is, Read

\footnotetext{
12 Iemhoff's formulation is somewhat ambiguous as to whether it applies to Kripke models or not; in view of McCarty's results, it seems permissible to assume that her formulation does apply here.

13 Veldman (1976), for example, introduced so-called 'modified Kripke models' that do allow for a completeness result but are not as natural as standard Kripke models.

14 It was suggested to me that intuitionistic logic could be saved by just using standard Tarski models in the intuitionistic meta-theory. However, note that it is classically false that Tarski models are complete with respect to intuitionistic logic (because they are complete for classical logic). Therefore, an intuitionistic theory proving that intuitionistic logic is complete with respect to Tarski models must be incompatible with classical logic. Hence, switching to intuitionistic logic in the meta-theory does not allow, ceteris paribus, to rescue intuitionistic logic as a correct logic by moving to Tarski models. Some additional assumption would be necessary. Also note that (extensions of) IZF and HAS will not do because, as McCarty (2008, Corollary 4) shows, "they prove that propositional and predicate intuitionistic logics are incomplete for Tarski semantics'.
} 
establishes that Beall and Restall's GTT leads to monism under the assumption of a relevance meta-logic.

Note that Read's critique of the 'insensitivity' of classical meta-logic can be interpreted as a worry about the coherence of pluralist views. Read does not accept the conclusions of both relevance and classical meta-logic, i.e. he implicitly endorses absoluteness.

Assuming that all parties are right, this example shows that the same set of desiderata may give rise to monism with a relevant meta-logic, pluralism with a classical metalogic, and a pluralism of potentially lesser extent with an intuitionistic meta-logic. The logics endorsed by a conception thus depend not only on the desiderata but also on the meta-logic.

For another example, Shapiro describes the following conflict. Tennant proved that his 'core logic' has a certain meta-mathematical property that allows him to argue that there is an 'epistemic gain in using his system' (2014, p. 204). However, Burgess claims that this proof makes crucial use of classical logic. But classical logic is not correct for Tennant. Shapiro resolves the conflict as follows, 'From the present, eclectic perspective [...] there is no need to adjudicate the dispute between Tennant and Burgess. For the eclectic logician, any of the established logics can be used to prove metatheoretic results about any of the established logics' (2014, p. 204 ). It is a fact that object-logics have different properties in different meta-logical contexts. There is no right meta-logical context.

What do these examples show? No matter which conception of 'correct logic' we subscribe to, there will be no fact of the matter, independent of meta-logic, about which logics can be considered correct. The case-study illustrates that the correct logics of sufficiently technical conceptions will depend on the meta-logic because even seemingly innocent mathematical properties of (object-)logics-such as their definition, consistency or completeness-rely heavily on the meta-logic and mathematical meta-theory. In conclusion, it seems that the absoluteness of correct logic underlying the coherence-worry of section 4.3 is not natural at all. If we accept naturalism in taking seriously the facts of mathematics, we cannot accept the absoluteness of correct logic.

\subsection{Contextualisation of correct logic}

Where are we at? Let's take stock. I argued in section 3 that conceptions of correct logic give meaning to 'correct logic' by specifying desiderata a logic has to satisfy to count as correct. In the previous sections 4.2 and 5.1, I showed-through reasoning by example - that a single conception of correct logic may give different verdicts about which logics count as correct in different meta-logical contexts because one and the same desideratum might be evaluated differently in different meta-logical contexts. These facts in conjunction suggest that the following principle is a better explanation of 'correct logic' than the absoluteness of correct logic from section 4.3:

Contextualisation of correct logic. Whether a logic is correct depends on the meta-logical context. 
As I argued in section 4.1, determining whether a logic is correct requires a meta-logic. The meta-logic, in turn, influences which logics are correct. Contextualised pluralism embraces these facts and thereby follows Caret's (2019) 'honest naturalism'. Contextualism is not unheard-of in the pluralist camp: Hjortland (2013), Shapiro (2014) and Caret (2017) suggest contextualism to understand 'valid' across object-logics. I suggest contextualism to understand 'correct logic' across meta-logics: the truth of sentences involving the unary predicate ' $x$ is a correct logic' depends on the sentence's meta-logical context, independently so of the conception of correct logic. ${ }^{15}$

The meta-logical context of a sentence is given by the (implicit or explicit) metalogic and (implicit or explicit) meta-logical assumptions of the conversation, debate, mathematical proof, research paper, discipline, or similar, in the context of which the sentence is uttered. Let's consider a few examples. I think it is uncontroversial that contemporary mathematical logic endorses a classical meta-logic. For this reason, assertions made in this discipline have a meta-logical context consisting of classical logic and possibly some set-theoretic assertions (even though this is often not explicitly acknowledged). For another example, imagine yourself in a course on intuitionistic mathematics in which the lecturer asserts that they will be using intuitionistic logic to establish certain meta-mathematical results. In this situation, their utterances will have a meta-logical context consisting of intuitionistic logic. I will further discuss how meta-logical contexts are determined through meta-linguistic negotiation in section 5.4 .

I will now discuss the nature of the proposed contextualism in more detail. Adapting from MacFarlane (2009, p. 232), I will say that an expression is (meta-logic-)indexical if and only if its content depends on the meta-logical context. Moreover, an expression is (meta-logic-)context-sensitive if and only if its extension depends on the meta-logical context. What kind of contextualism are we dealing with here? It turns out that there are two cases depending on the meta-logical meaning-variance worry as discussed in section 4.2: if the meaning of logical connectives changes and, accordingly, the meaning of the desiderata changes with meta-logic, then the desiderata cannot describe an absolute meaning of 'correct logic'. We are left, instead, with a bunch of different meanings of 'correct logic'.

First, suppose that we are dealing with a conception of correct logic for which the meaning-variance worry is serious. This means that the meaning of the desiderata employed varies so much that it is unclear whether they give rise to a stable notion of correct logic across diverse meta-logics. In this situation, we end up with an indexical contextualism because the propositional content of 'correct logic' depends on the meta-logical context. Inspired by Kaplan (1979) (and Hjortland 2013, p. 361), I suggest to understand 'correct logic' in terms of its character, i.e. a function from contexts to contents. Accordingly, the character of 'correct logic' is the function that maps a logic $\mathcal{L}_{\text {meta }}$ to the propositional content expressed by the desiderata in the given meta-logical context. If we write $P_{i}^{\mathcal{L}}$ to denote the desideratum $P_{i}$ interpreted in the meta-logic $\mathcal{L}$,

\footnotetext{
15 This contextualisation is not restricted to conceptions considering the absolute predicate of ' $x$ a correct logic' but also works for instrumentalist views endorsing binary relations, such as ' $x$ is a correct logic for purpose/application $y^{\prime}$.
} 
then we can give the following schematic representation of the character:

$$
\begin{aligned}
& c_{\text {correct logic }}: \text { meta-logical contexts } \rightarrow \text { propositions } \\
& \mathcal{L}_{\text {meta }} \mapsto P_{0}^{\mathcal{L}_{\text {meta }}}(\cdot) \wedge \ldots \wedge P_{n}^{\mathcal{L}_{\text {meta }}(\cdot)}
\end{aligned}
$$

In conclusion, the phrase ' $L$ is a correct logic' means that ' $L$ satisfies $P_{0}$-inintuitionistic-logic and $L$ satisfies $P_{1}$-in-intuitionistic-logic and so on' in an intuitionistic meta-logical context. In a classical meta-logical context, it means ' $L$ satisfies $P_{0}$-in-classical-logic and $L$ satisfies $P_{1}$-in-classical-logic and so on'. This is indexical contextualism because the propositional content of 'correct logic' changes: in virtue of the meaning-variance worry, $P_{i}$-in-classical-logic and $P_{i}$-in-intuitionistic-logic cannot be treated as having the same meaning but rather as distinct predicates with distinct meanings.

For the indexical contextualist, the meaning of 'correct logic' is not fixed by the intension or extension of the desiderata but by the character of 'correct logic'. This character determines the meaning of 'correct logic' and thereby solves the meaningvariance-worry. The meaning of related notions, such as validity-in-correct-logic, can be derived from the character of 'correct logic'. In this way, indexical contextualism takes care of the meta-logical meaning-variance worry.

Let's now move to the second case and assume that we are dealing with a conception of correct logic for which the meaning-variance worry is not serious. In this case, the meaning of the desiderata is stable enough across meta-logic to give rise to a single intension-the propositional content of 'correct logic'. As we have seen above, the extension of 'correct logic' depends on the meta-logical context. For this reason, we end up with a non-indexical contextualism schematically illustrated as follows:

$$
\begin{aligned}
e_{\text {correct logic }}: \text { meta-logical contexts } & \rightarrow \text { classes of logics } \\
\mathcal{L}_{\text {meta }} & \mapsto\left\{\mathcal{L} \mid P_{0}(\mathcal{L}), \ldots, P_{n}(\mathcal{L}) \text { hold in } \mathcal{L}_{\text {meta }}\right\}
\end{aligned}
$$

In this case, the propositional content of 'correct logic' is the same at each metalogical context. However, the extension varies as expressed by the map $e_{\text {correct logic }}$ : the extension of 'correct logic' functionally depends on the meta-logical context.

A comparison to the case of contextualist treatments of knowledge might be helpful to appreciate the two forms of contextualism given here. What does it mean to know something? One way to treat the intuition that 'know' has different meanings in different situation is epistemic contextualism: MacFarlane describes its standard view as taking 'know' to be indexical, 'knowledge-attributing sentences express different propositions at different contexts of use' (2009, p. 236). Against this view, he proposes a non-indexical contextualism where ' $x$ knows $y$ ' is the same relation at every context but "truth values of sentences containing "know" depend on the epistemic standard in play at the context of use, not because this standard affects which proposition is expressed, but because it helps determine which circumstance of evaluation to look at in deciding whether these sentences are true or false at the context' (2009, p. 237). The situation concerning 'correct logic' is comparable. If a given conception of correct logic is affected by the meaning-variance worry, then the indexical 
contextualism put forward above attributes different propositions to 'correct logic' in different meta-logical contexts. On the other hand, if the meaning-variance worry is not serious, then, by the non-indexical contextualism proposed above, the proposition expressed by 'correct logic' does not depend on the meta-logical context. However, the meta-logical context determines a standard of evaluation according to which truth values of sentences containing 'correct logic' are determined. ${ }^{16}$

Both ways of contextualising solve the coherence-worry. I argued in section 4.3 that the coherence-worry of logical pluralism is based on the absoluteness of correct logic, i.e. the negation of contextualisation. So, by endorsing contextualisation, the pluralist avoids these and further coherence issues. For the contextualising pluralist, the meaning of 'correct logic' is decoupled from any specific meta-logic. Contextualised pluralism does not have a meta-logic that needs to be justified. In a given meta-logical context, there is nothing to be justified: we just are in this context.

I argued that contextualisation has naturalistic appeal and solves both the coherenceworry and the meaning-variance-worry. In the next section, I discuss contextualism and proper pluralism.

\subsection{Contextualisation and pluralism proper}

Is contextualised pluralism still proper pluralism? Let me first emphasise again that contextualisation is a supplement for any given pluralist conception and does not form a conception itself. This means that if we considered the original conception to be proper in the sense of section 2, then I have to ensure that the corresponding contextualised conception is proper as well. So why would we accept that the original conception is proper? We would accept this in virtue of an argument. That argument might be explicitly deductive, abductive, or rather informal. In any case, as I have argued in section 4.1, the meta-logic matters for this argument, i.e. the argument that the original conception is proper can only be deemed valid in virtue of its meta-logical context. Of course, if the argument was valid before explicitly acknowledging its meta-logical context, then it will still be valid when one explicitly acknowledges its meta-logical context. It will still show a proper disagreement about the validity of an argument. This disagreement does not disappear because its meta-logical context is acknowledged.

However, it could happen that there are meta-logical contexts without disagreement, or in which there is at most one correct logic. Would this be a problem? I do not think so. The crucial point is that contextualisation does not weaken the conception it amends. The same argument still shows that the conception is proper in its metalogical context. But this context is nothing new, it was always there and necessary to conduct the argument in the first place. If you observe that the properness-argument is not valid in a different meta-logical context, then this was already the case for the original conception. Under the original conception, it was just not acknowledged that this is the case.

\footnotetext{
16 Note that this is even more intricate than in MacFarlane's proposal because the meta-logical context could allow more truth values than just true and false.
} 
This situation compares to Steinberger's conclusion that 'if logic is normative, competition between logics may be inevitable' (2019, p. 17) for the pluralist. The essential point is that it will require more care and more work for the pluralist to account for the normativity of logic than it does for the monist. But that's not a bug, it's a feature. If proper pluralism is true, then we have to live with equally correct logics disagreeing about the validity of an argument. That's the point of pluralism. From such a pluralistic point of view, it would seem rather odd if different logics did not disagree about 'correct logic'. All this shows is that pluralists must not only account for different judgements of whether or not an argument is valid but also explain diverging judgements about which logics are correct. Competition between meta-logics may be inevitable. Contextualism explains this competition.

Of course, all this is not to say that once contextualism is adopted, there are no reasons to consider stronger requirements for pluralism proper. Maybe the pluralists want disagreement in several meta-logical contexts that are particularly relevant for them. Or maybe disagreement in one particular meta-logic is more important than disagreement in other meta-logics. Contextualisation might thus be taken as yet another reason to refine what makes pluralism proper. For now, my conclusion is just that a contextualised pluralist conception is just as proper as the original conception was.

\subsection{Contextualisation in practice}

I showed above that the pluralism of Beall and Restall varies with meta-logic because (parts of) their argument are not valid in non-classical meta-logics. Naturalism requires to take these facts of mathematics seriously, and I argued that contextualisation explains them better than relying on the absoluteness of 'correct logic'. However, if I take naturalism seriously, there is an other piece of evidence that I cannot ignore: an overwhelming majority of contemporary logicians, even those working on non-classical logics, rely on classical logic as their all-purpose meta-logic. So, from this sociological point of view, there does not seem to be much support for non-classical meta-logics. If contextualism is true, shouldn't we find evidence for it also in the practice of logic? To try and alleviate this objection, I will first argue that this practice is coherent with contextualisation, and then try to given an explanation why such a phenomenon is to be expected nevertheless.

Contextualisation does not prescribe that all meta-logics must be given equal weight but only states that the truth of statements such as ' $\mathcal{L}$ is a correct logic' depends on the meta-logical context. I do not wish to police how logicians proceed and judge their choices. The only point I'm making is that their results about logics depend on their meta-logic. These meta-logical commitments might be made implicitly, e.g. when Beall and Restall rely on the completeness of intuitionistic logic with respect to Kripke semantics for their argument, or explicitly, e.g. when Read changes the meta-logic to reinterpret Beall and Restall's desiderata. A meta-logical commitment is unavoidable.

Considering these circumstances, it is not surprising that there is a prevalent metalogic, viz. classical logic, used in contemporary logic. There is a dialectic need for a common meta-logic. This situation can best be understood through Kouri Kissel's 
(2019) framework of meta-linguistic negotiations in logic. Her idea is this: imagine that an intuitionistic logician and a classical logician are discussing a proof. Let's also assume that they are not aware of each other's faith, i.e. they do not know of each other that they ascribe different meanings to 'or' and 'not'. If they reach a disagreement during their discussion, it is typically interpreted as a verbal disagreement that should disappear once the participants realise that it is only in virtue of them ascribing different meanings to the logical connectives. Kouri Kissel, however, suggests that we should understand such disagreements as a meta-linguistic negotiation of the meaning of the connectives. So when the classical and intuitionistic logicians disagree about the validity of the law of excluded middle, then they are not just talking past each other but in fact are making moves in a negotiation on the meanings of the logical connectives.

This framework perfectly applies to the dialectic of meta-logical considerations. Let me explain this with my running example. When Beall and Restall (2005, Chapter 6) invoke the completeness of intuitionistic logic with respect to Kripke models, they make a move in the meta-linguistic negotiation of the meta-logical framework (settling their meta-logical context). This is because the completeness theorem is not meta-logically innocent: as I explained above, this completeness entails certain meta-theoretical commitments that result in valid meta-logical principles beyond pure intuitionistic logic. So even if you are reluctant to explicitly specify your meta-logic, by accepting certain mathematical tools into your reasoning you might make implicit moves towards determining your meta-logic. Just like the intuitionistic and classical logicians negotiate their logic when they discuss the validity of a proof, contemporary logicians meta-linguistically negotiate the meta-logic when they argue about correct logics. But then, as I mentioned above, there does not seem to be much negotiation going on in contemporary discussions of correct logic.

Combining Kouri Kissel's framework with a contextualist understanding of metalogic allows us to explain the prevalent use of classical logic as meta-logic as follows. There is a need for meta-logic. The meta-logic is negotiated between participants of the debate on correct logic, or, more generally, between contemporary logicians who study (properties of) logics, i.e. use some logic to study logic(s). To participate in this debate and engage with other logicians' work, it is thus necessary to establish a common ground. More often than not, this common ground is classical logic. This might be because classical logic might have certain properties which make it convincing as a meta-logic to study other logics. For example, in comparison to intuitionistic logic, it seems that working in classical logic is a bit easier-classical logic is stronger and therefore allows you to do more, and often to do the same but quicker. Connected to this is the historical fact that most mathematical tools for the study of logics have been developed in classical logic. Changing to a different meta-logic would thus require to give up on many established results (such as the completeness of intuitionistic logic with respect to Kripke models) and require to develop new tools. In this sense, relying on classical meta-logic is also a convenient choice if one wants to get to the core of the matter without redeveloping other results. It will, therefore, be much less tempting to employ a non-classical meta-logic because when a logician wants to compare their results to those of a peer, then our logician might first have to redevelop their results in a deviant meta-logic (if that is even possible). Clearly, this does not mean that it is never 
done: as explained above, Read (2006) does change the meta-logic in his critique of Beall and Restall; he explicitly disagrees on their meta-logical commitments.

While classical logic is the lingua franca of contemporary logic, it did not necessarily have to be that way. The facts of logic are indisputable but the practice of logic might just be different. Just like this paper could have been written in Swahili instead of English, it is conceivable that logic could be practised with a non-classical meta-logic as lingua franca and semantical tools for logical analysis that we have not yet discovered.

\subsection{Contextualising Beall and Restall}

Let's contextualise Beall-Restall-pluralism. The resulting conception endorses classical, intuitionistic and relevance logic in a classical meta-logic. If I am correct, it does not (uncontentiously) support intuitionistic logic in an intuitionistic meta-logic. If Read is right, then relevance logic is the only correct logic in a relevance meta-logic. If Beall-Restall-pluralism is proper, then so is the resulting conception as the argument carries over in - at least - the classical context. And if we are uncertain about the meta-logical context, all of this gives us reasons to believe that classical, intuitionistic and relevance logics are correct.

Before wrapping up, I would like to briefly suggest that the contextualising pluralist might have an advantage over the absolute pluralist, monist or nihilist in terms of 'common ground'. If Beall and Restall are absolutist, then they must disagree with Read. However, if they contextualise, then they allow for common ground with him: they could accept that his argument is correct in a relevance context. They could then point out that his perspective is not comprehensive enough. In this way, contextualisation allows for combining seemingly opposing arguments into one conception. If and how this approach could convince a monist is beyond the scope of this paper.

\section{Conclusions and outlook}

Should pluralists be pluralists about pluralism? I think they should, in the form of contextualism about correct logic. This contextualism has naturalistic appeal. Moreover, it seems intrinsically pluralistic to accept that the extent of 'correct logic' will vary with meta-logic. Meta-logic cannot be discarded from any reasoning about nihilist, monist or pluralist conceptions of logic. I identified two worries for the pluralist's use of meta-logic: the coherence-worry and the meaning-variance-worry; and I argued that contextualism about correct logic allows the pluralist to solve these worries. If pluralism is true, we might have to accept that we can give a precise collection of correct logics only relative to a given meta-logic. But that's not a bug-it's a feature! We are then pluralists about the extent of our pluralism.

I arrived at contextualism through an analysis of logical pluralism. But what happens if we assume contextualism first and then consider the debate between monists and pluralists? From a contextualist point of view, it does not seem very natural that there should be just one correct logic-One True Logic. This would require, in all meta- 
logical contexts, an argument that this particular logic is the only logic satisfying the monist's desiderata. It seems, therefore, conceivable that monists might have to reject the contextualisation of 'correct logic'. I argued above that naturalism favours contextualism. In this sense, contextualism might put pressure on monists to justify this absoluteness without begging the question by appealing to their One True Logic.

The full ramifications of a contextualist view on 'correct logic' for the debate between monists and pluralists are still to be worked out. Yet, I believe that there are good reasons that the resulting picture supports the pluralist. There are two interrelated questions here: how many logics are correct? And, which logics are correct? While contextualism about correct logic makes answering the second question intricate, it seems that a pluralist view better explains contextualism than logical monism would. This contextualisation, if true, might require a redefinition of logical pluralism: many logics are correct—but need they be correct at the same time in the same context? Or is it enough that there are at least two distinct logics which are correct in different contexts? In any case, this discussion shows that the question of whether logical pluralism is true should be uncoupled from the question of exactly which logics are true.

Acknowledgements I am very thankful for many helpful discussions with Owen Griffiths; his feedback highly improved this paper. I thank Leon Commandeur for his very helpful comments on several drafts of this paper. Audiences at the Bergen Logic Group and the Set Theory in Hamburg-Amsterdam-Cambridge seminar provided helpful feedback and challenging questions. I am grateful for two anonymous referees whose motivating feedback helped me improving this paper.

Funding This research was supported by a Cultuurfondsbeurs of the Prins Bernhard Cultuurfonds (Reimande Bas Fonds and Niemans-Schootemeijer Fonds) as well as a doctoral scholarship of the Studienstiftung des deutschen Volkes.

Open Access This article is licensed under a Creative Commons Attribution 4.0 International License, which permits use, sharing, adaptation, distribution and reproduction in any medium or format, as long as you give appropriate credit to the original author(s) and the source, provide a link to the Creative Commons licence, and indicate if changes were made. The images or other third party material in this article are included in the article's Creative Commons licence, unless indicated otherwise in a credit line to the material. If material is not included in the article's Creative Commons licence and your intended use is not permitted by statutory regulation or exceeds the permitted use, you will need to obtain permission directly from the copyright holder. To view a copy of this licence, visit http://creativecommons.org/licenses/by/4.0/.

\section{References}

Beall, J., \& Restall, G. (2005). Logical pluralism. Oxford University Press.

Caret, C. R. (2017). The collapse of logical pluralism has been greatly exaggerated. Erkenntnis, 82(4), 739-760. https://doi.org/10.1007/s10670-016-9841-7

Caret, C. R. (2019). Why logical pluralism? Synthese. https://doi.org/10.1007/s11229-019-02132-w

Dummett, M. (1975). The philosophical basis of intuitionistic logic. In M. Dummett (Ed.), Truth and other enigmas (pp. 215-247). Cambridge: Harvard UP.

Dummett, M. (1977). Elements of Intuitionism. Oxford University Press.

Griffiths, O. (2013). Problems for logical pluralism. History and Philosophy of Logic, 34(2), 170-182. https://doi.org/10.1080/01445340.2012.749030

Griffiths, O., \& Paseau, A. (2021). The instability of logical pluralism (unpublished manuscript).

Hjortland, O. (2013). Logical pluralism, meaning-variance, and verbal disputes. Australasian Journal of Philosophy, 91(2), 355-373. https://doi.org/10.1080/00048402.2011.648945 
Iemhoff, R. (2020). Intuitionism in the philosophy of mathematics. In E. N. Zalta (Ed.), The Stanford encyclopedia of philosophy, fall (2020th ed.). Metaphysics Research Lab: Stanford University.

Jech, T. (1966). On ordering of cardinalities. Bulletin de l'Académie Polonaise des Sciences Série des Sciences Mathématiques, Astronomiques et Physiques, 14, 293-296 (loose addendum).

Kaplan, D. (1979). On the logic of demonstratives. Journal of Philosophical Logic, 8(1), 81-98. https:// doi.org/10.1007/BF00258420

Keefe, R. (2014). What logical pluralism cannot be. Synthese, 191(7), 1375-1390. https://doi.org/10.1007/ s11229-013-0333-x

Kouri Kissel, T. (2019). Metalinguistic negotiation and logical pluralism. Synthese. https://doi.org/10.1007/ s11229-019-02264-z

Kreisel, G. (1962). On weak completeness of intuitionistic predicate logic. Journal of Symbolic Logic, 27(2), 139-158. https://doi.org/10.2307/2964110

Lubarsky, R. S., \& Rathjen, M. (2008). On the constructive Dedekind reals. Logic and Analysis, 1(2), 131-152. https://doi.org/10.1007/s11813-007-0005-6

MacFarlane, J. (2009). Nonindexical contextualism. Synthese, 166(2), 231-250. https://doi.org/10.1007/ s11229-007-9286-2

Martin, B., \& Hjortland, O. (2021). Logical predictivism. Journal of Philosophical Logic, 50(2), $285-318$. https://doi.org/10.1007/s10992-020-09566-5

McCarty, C. (2008). Completeness and incompleteness for intuitionistic logic. Journal of Symbolic Logic, 73(4), 1315-1327. https://doi.org/10.2178/jsl/1230396921

Paseau, A. (2007). Review: Logical pluralism. Mind, 116(462), 391-396. https://doi.org/10.1093/mind/ fzm391

Priest, G. (2001). Logic: One or many? In J. Woods \& B. Brown (Eds.), Logical consequences: Rival approaches (pp. 23-38). Hermes Scientific Publishers.

Priest, G. (2005). Doubt truth to be a liar. Oxford University Press.

Priest, G. (2014). Revising logic. In: P. Rush (Ed.), The metaphysics of logic, chap. 12. Cambridge University Press. https://doi.org/10.1017/CBO9781139626279

Quine, W. V. O. (1986). Philosophy of logic. Harvard University Press.

Read, S. (2006). Monism: The one true logic. In: D. DeVidi, \& T. Kenyon (Eds.), A logical approach to philosophy: Essays in memory of Graham Solomon, The Western Ontario series in philosophy of science (Vol. 69, pp. 193-209). Springer. https://doi.org/10.1007/1-4020-4054-7_10

Russell, G. (2018). Logical nihilism: Could there be no logic? Philosophical Issues, 28(1), 308-324. https:// doi.org/10.1111/phis.12127

Russell, G. (2021). Logical pluralism. In E. N. Zalta (Ed.), The Stanford encyclopedia of philosophy, summer (2021st ed.). Metaphysics Research Lab: Stanford University.

Sereni, A., \& Fogliani, M. P. S. (2017). How to water a thousand flowers. On the logic of logical pluralism. Inquiry: An Interdisciplinary Journal of Philosophy. https://doi.org/10.1080/0020174X.2017. 1370064

Shapiro, S. (2000). The status of logic. In: P. Boghossian, \& C. Peacocke (Eds.), New essays on the a priori (pp. 333-338). Oxford University Press.

Shapiro, S. (2014). Varieties of logic. Oxford University Press.

Skvortsov, D. (1998). On some Kripke complete and Kripke incomplete intermediate predicate logics. Studia Logica: An International Journal for Symbolic Logic, 61(2), 281-292.

Steinberger, F. (2019). Logical pluralism and logical normativity. Philosophers' Imprint 19.

Veldman, W. (1976). An intuitiomstic completeness theorem for intuitionistic predicate logic. Journal of Symbolic Logic, 41(1), 159-166. https://doi.org/10.1017/s0022481200051859

Williamson, T. (2014). Logic, metalogic and neutrality. Erkenntnis, 79, 211-231. https://doi.org/10.1007/ s10670-013-9474-z

Woods, J. (2019). Logical partisanhood. Philosophical Studies, 176(5), 1203-1224. https://doi.org/10.1007/ s11098-018-1054-2

Wright, C. (1986). Inventing logical necessity. In J. Butterfield (Ed.), Language, mind and logic. Cambridge University Press.

Publisher's Note Springer Nature remains neutral with regard to jurisdictional claims in published maps and institutional affiliations. 